\title{
Huge portal venous aneurysm incidentaloma caused by intrahepatic arterioportal fistula accompany with hepatobiliary stones and cholangitis
}

\author{
Zheng-Hao Liu ${ }^{1,2,3,4,5}$, Dong-Kai Zhou ${ }^{1,2,3,4,5}$, Yu-Cheng Xiang ${ }^{1,2,3,4,5}$, Cheng Zeng ${ }^{1,2,3,4,5}$, Wei-Lin Wang ${ }^{1,2,3,4,5}$ \\ ${ }^{1}$ Department of Hepatobiliary and Pancreatic Surgery, The Second Affiliated Hospital, Zhejiang University School of Medicine, Hangzhou, China; \\ ${ }^{2}$ Key Laboratory of Precision Diagnosis and Treatment for Hepatobiliary and Pancreatic Tumor of Zhejiang Province, Hangzhou, China; ${ }^{3}$ Research \\ Center of Diagnosis and Treatment Technology for Hepatocellular Carcinoma of Zhejiang Province, Hangzhou, China; ${ }^{4} \mathrm{Clinical}$ Medicine \\ Innovation Center of Precision Diagnosis and Treatment for Hepatobiliary and Pancreatic Disease of Zhejiang University, Hangzhou, China; \\ ${ }^{5}$ Clinical Research Center of Hepatobiliary and Pancreatic Diseases of Zhejiang Province, Hangzhou, China \\ Correspondence to: Weilin Wang. Department of Hepatobiliary and Pancreatic Surgery, The Second Affiliated Hospital, Zhejiang University School of \\ Medicine, No. 88 Jiefang Road, Hangzhou 310009, China. Email: wam@zju.edu.cn.
}

Submitted Aug 16, 2020. Accepted for publication Sep 07, 2020.

doi: $10.21037 / \mathrm{hbsn}-20-637$

View this article at: http://dx.doi.org/10.21037/hbsn-20-637

A 64-year-old female was admitted to our department because of abdominal discomfort and recurrent fever for 1 month, she had attended her local hospital 10 days earlier, where the sign of fever was regarded as being due to the pulmonary infection, however, moxifloxacin and peramivir had no therapeutic effect. On admission, the physical examinations were showed an upper abdominal thrill and auscultation heard a blood vessel murmur at the same location, lab tests were not showed any remarkable results, the tumor marker levels were within normal range. The portal color contrast ultrasonography was showed a $5.4 \mathrm{~cm}$ $\times 3.3 \mathrm{~cm}$ bright spot in the left intrahepatic bile duct, and a $0.9 \mathrm{~cm}$ distal bright spot in, and $0.7 \mathrm{~cm}$ expansion of the right intrahepatic bile duct (Figure 1A). Contrast-enhanced computed tomography (CT) were showed intrahepatic cholangiectasis, tumor-like dilatation of the left portal ramus, and left liver atrophy and cirrhosis (Figure 1B). Magnetic resonance cholangiopancreatography (MRCP) were showed a patchy $5.1 \mathrm{~cm} \times 3.8 \mathrm{~cm}$ T2W1 hyperfocal lesion in the left lobe of the liver that was compressing the adjacent bile duct, leading to dilation and infection (Figure 1C,D). The endoscopic was showed chronic gastritis with mild esophageal varices. A laparotomy was performed which a left hemihepatectomy was done after MDT consultation was made. During the surgery, the left liver was atrophied with cirrhotic nodules were noticed. In the first hilum of the liver, a $6 \mathrm{~cm} \times 5 \mathrm{~cm}$ incidentaloma caused by arteriovenous fistula was confirmed, which lies between the left hepatic artery and the sagittal part of the portal vein, dilatation of the proper hepatic artery and the left hepatic artery were observed (diameter of both, $1 \mathrm{~cm}$ ). The perigastric veins dilatation and spleen enlargement were noticed, Arteriovenous fistula was clearly visible in the resected lesion (Figure 1E), the adjacent bile duct dilation was seen with stones inside the resected sample's bile ducts (figure was not shown). A pathology examination showed that the intrahepatic bile duct of the left liver was dilated, and numerous neutrophils were seen around the intrahepatic bile ducts (Figure $1 F$ ), arteriovenous fistula was confirmed (Figure 1G), liver cirrhosis was noticed with small nodules (figure was not shown). After the surgery, the fever was resolved and the patient was discharged from hospital at 3 weeks postoperatively.

An arterioportal fistula is a functional or organic communication between the portal venous system and the hepatic arterial branch, and is an infrequent cause of presinusoidal portal hypertension. Intrahepatic arterioportal fistulas (IAPFs) may be caused by trauma, malignant tumor (1), or iatrogenic injury, while some cases are hereditary (2). To our knowledge, this is the first report in the English language literature of IAPF caused incidentaloma with intrahepatic stones and cholangitis.

The symptomatology of IAPF encompasses hepatomegaly, portal hypertension bleeding episodes, ascites, and 

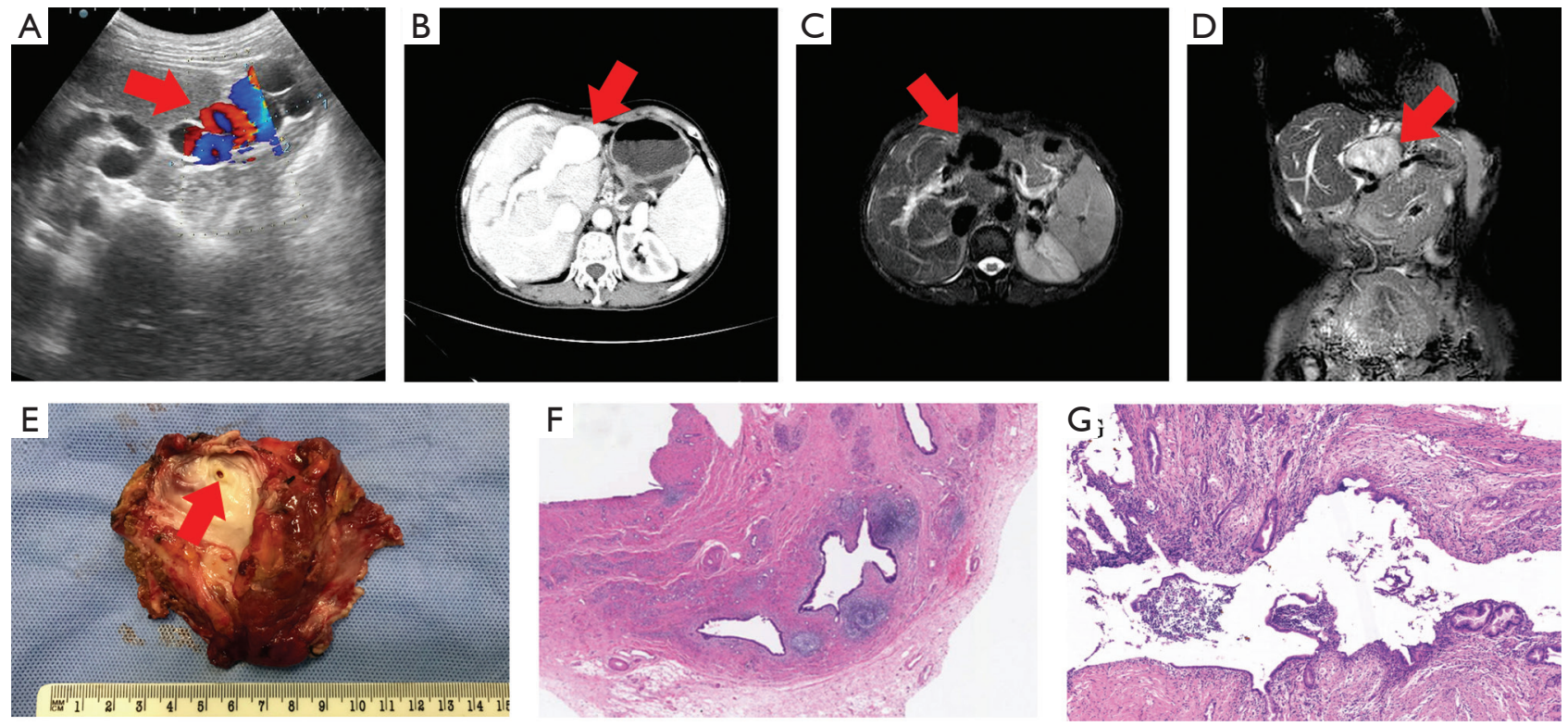

Figure 1 Imaging diagnosis, specimen and pathology. (A,B,C,D) Imaging diagnosis. (A) Color Doppler image reveals multidirectional flow. (B) Earlier enhancement of the portal vein during the arterial phase. Axial- (C) and coronal-reformatted (D) magnetic resonance cholangiopancreatography (MRCP) reveals tumor-like dilation of the left portal vein. (E) Specimen, the arrow points to the arteriovenous fistula. (F,G) Pathology. Histological staining showing numerous neutrophils around intrahepatic bile duct $(\mathrm{F}, \times 20)$ and arteriovenous fistula $(\mathrm{G}, \times 40)$.

splenomegaly. In some typical arterioportal fistula (APF) cases, a characteristic bruit can be heard and a touch tremor is present in the right upper quadrant. In addition, small IAPFs can be asymptomatic. the patient's primary symptom, recurrent fever without obvious right upper abdominal pain, resulted in misdiagnosis as pneumonia at a local hospital. The patient's symptoms of recurrent fever were caused by cholangitis due to the presence of bile-duct stones, the bile duct dilation could due to the IAPF caused incidentaloma which were confirmed by surgery.

In patients with liver cirrhosis, the frequency of nontumorous APFs is very high and such individuals are at increased risk of hepatocellular carcinoma (HCC) (3). However, distinguishing between APFs and tumors can be difficult (4). Identifying a tumorous APF near a minor HCC by CT is hampered by the fact that the contrast agent highlights the entire APF area during the hepatic arterial phase (HAP). Generally, wedge-shaped enhancement of the normal vessels passing through the lesion indicates a non-tumorous APF. Moreover, HCC manifests as an area of heterogeneous low attenuation, but the magnitude of attenuation of nontumorous APFs is not lower than that of adjacent liver parenchyma. The equilibrium phase is most useful for differentiating between HCC and APFs (3).
For the imaging result in this patient, on Doppler ultrasound (US) images of the patients showed with IAPF, the velocities in the hepatic vein and inferior vena cava were intensified due to entry of arterial blood into the venous system. On dynamic CT, IAFPs were result in enhancement of the peripheral portal vein branches before the central portal vein $(3,5)$ and enhancement of the hepatic vein in the arterial phase. The findings of dynamic MRI were typically similar to those of dynamic CT.

For the treatment of liver cirrhosis and portal hypertension in this case, due to the surgery was completed resect the lesion and stopped the abnormal vascular communication, the portal flow and pressure will be greatly decreased postoperative time, there was no evidence showed hypersplenism which must need surgical intervention, so no more interventional operative procedure will be applied in this case. The patient did not show any symptom caused by portal hypertension during the follow up period, the chronic gastritis was also recovered to normal situation.

The optimum therapy for APF remains controversial, it can be treated by hepatic artery ligation (6), partial hepatectomy (7), percutaneous transarterial embolization (8), and liver transplantation (9). Endovascular techniques are considered the first line options (10), but interventional 
therapy may not be suitable for $>8 \mathrm{~mm}$ APFs with a high flow rate because of the risk of arterial thrombosis, movement of the metallic coil, and pulmonary embolism (8). In this case, interventional radiological treatment was not performed because of the large size of the fistula and bile duct stones, incidentaloma were simultaneously presented, instead the hemihepatectomy was done to resolve all of the problems after the MDT was made.

We described a case of a patient with incidentaloma caused by IAPF accompany with intrahepatic stones and cholangitis, the primary symptom of which was recurrent fever. The patient underwent a left hemihepatectomy and were not experienced recurrence of fever during the 6-month follow up. Therefore, Long-term incidentaloma caused by IAPF could cause the presence of bile-duct dilation with stones and cholangitis.

\section{Acknowledgments}

Funding: None.

\section{Footnote}

Provenance and Peer Review: This article was a standard submission to the Hepatobiliary Surgery and Nutrition. The article did not undergo external peer review.

Conflicts of Interest: All authors have completed the ICMJE uniform disclosure form (available at https://hbsn. amegroups.com/article/view/10.21037/hbsn-20-637/coif). The authors have no conflicts of interest to declare.

Ethical Statement: The authors are accountable for all aspects of the work in ensuring that questions related to the accuracy or integrity of any part of the work are appropriately investigated and resolved. Written informed consent was obtained from the patient for publication of this manuscript and any accompanying images.

Open Access Statement: This is an Open Access article distributed in accordance with the Creative Commons Attribution-NonCommercial-NoDerivs 4.0 International License (CC BY-NC-ND 4.0), which permits the noncommercial replication and distribution of the article with the strict proviso that no changes or edits are made and the original work is properly cited (including links to both the formal publication through the relevant DOI and the license). See: https://creativecommons.org/licenses/by-nc-nd/4.0/.

\section{References}

1. Morse SS, Sniderman KW, Galloway S, et al. Hepatoma, arterioportal shunting, and hyperkinetic portal hypertension: therapeutic embolization. Radiology 1985;155:77-82.

2. Liu YR, Huang B, Yuan D, et al. Unusual case of digestive hemorrhage: celiac axis-portal vein arteriovenous fistula. World J Gastroenterol 2015;21:1362-4.

3. Choi BI, Lee KH, Han JK, et al. Hepatic arterioportal shunts: Dynamic CT and MR features. Korean Journal of Radiology 2002;3:1-15.

4. Yu JS, Kim KW, Sung KB, et al. Small arterial-portal venous shunts: a cause of pseudolesions at hepatic imaging. Radiology 1997;203:737-42.

5. Elsayes KM, Shaaban AM, Rothan SM, et al. A Comprehensive Approach to Hepatic Vascular Disease. Radiographics 2017;37:813-36.

6. Sutcliffe R, Mieli-Vergani G, Dhawan A, et al. A novel treatment of congenital hepatoportal arteriovenous fistula. J Pediatr Surg 2008;43:571-3.

7. Tannuri AC, Tannuri U, Lima FR, et al. Congenital intrahepatic arterioportal fistula presenting as severe undernutrition and chronic watery diarrhea in a 2-year-old girl. J Pediatr Surg 2009;44:e19-22.

8. Chen Q, Tack C, Morcos M, et al. Embolotherapy of an arterioportal fistula. Cardiovasc Intervent Radiol 2007;30:1047-51.

9. Heaton ND, Davenport M, Karani J, et al. Congenital hepatoportal arteriovenous fistula. Surgery 1995;117:170-4.

10. Kumar A, Ahuja CK, Vyas S, et al. Hepatic arteriovenous fistulae: role of interventional radiology. Dig Dis Sci 2012;57:2703-12.

Cite this article as: Liu ZH, Zhou DK, Xiang YC, Zeng C, Wang WL. Huge portal venous aneurysm incidentaloma caused by intrahepatic arterioportal fistula accompany with hepatobiliary stones and cholangitis. HepatoBiliary Surg Nutr 2021;10(2):288-290. doi: 10.21037/hbsn-20-637 